\title{
The function of ncRNAs in rheumatic diseases
}

\author{
Weilin Chen ${ }^{\ddagger 1}$, Di Liü,1, Quan-Zhen $\mathrm{Li}^{1}$ \& Honglin Zhu*,1 \\ ${ }^{1}$ Department of Rheumatology, Xiangya Hospital, Central South University, 87 Xiangya Road, Changsha, Hunan 410008, PR China \\ *Author for correspondence: Tel.: +86 7318975 3548; Fax: +86 7318975 3548; honglinzhu@csu.edu.cn \\ $¥$ Authors contributed equally
}

Rheumatic diseases are a group of chronic heterogeneous autoimmune disorders characterized by abnormal regulation of the innate and adaptive immune systems. Despite extensive efforts, the full spectrum of molecular factors that contribute to the pathogenesis of rheumatic diseases remains unclear. ncRNAs can govern gene expression at the transcriptional and post-transcriptional levels in multiple diseases. Recent studies have demonstrated an important role for ncRNAs, such as miRNAs and IncRNAs, in the development of immune cells and rheumatic diseases. Here, we focus on the epigenetic regulatory roles of ncRNAs in the pathogenesis of rheumatic diseases and as biomarkers of disease state.

First draft submitted: 22 August 2018; Accepted for publication: 5 February 2019; Published online: 7 June 2019

Keywords: circular RNA •IncRNA • miRNA • ncRNA • piRNAs • rheumatic diseases

Rheumatic diseases are chronic autoimmune inflammatory disorders caused by perturbations of the innate and adaptive immune systems and of cellular activity, which result in substantial inflammatory responses and tissue damage. The main rheumatic diseases include rheumatoid arthritis (RA), systemic lupus erythematosus (SLE), idiopathic inflammatory myopathy (IIM), systemic sclerosis (SSc) and Sjögren's syndrome (pSS). The pathogenesis of rheumatic diseases remains unclear despite great advances in recent years. Both genetic and environmental factors contribute to these disorders, and the importance of epigenetics, including methylation and histone modification, and ncRNAs has become increasingly apparent [1,2].

ncRNAs are a class of nonprotein-coding RNAs, which can be divided into housekeeping RNAs and regulatory RNAs. Housekeeping RNAs are involved in ribosomal machinery and include rRNAs, snRNAs, snoRNAs and tRNAs. Regulatory RNAs include short ncRNAs (<200 nucleotides; miRNAs and piRNAs) and lncRNAs (longer than 200 nucleotides). ncRNAs play important roles in innate and adaptive immunity and inflammation. The most commonly studied ncRNAs in rheumatology are miRNAs and lncRNAs. Here, we summarize the most current knowledge and existing data on ncRNAs as causal factors of the pathogenesis of rheumatic diseases and as biomarkers of disease state.

\section{The biology of ncRNAs in inflammation \& rheumatic disease}

Since the discovery of the first miRNA in 1993, studies on ncRNAs in autoimmune diseases and inflammation have boomed [3]. Increasing evidence has shown that dysregulated ncRNAs, especially miRNAs can influence multiple biological processes, such as immune homeostasis, immune tolerance, immune cell development, differentiation and proliferation, and the imbalance of proinflammatory/anti-inflammatory cytokines [4-6]. IncRNAs played important roles in immune system, such as regulating the expression of IFN- $\gamma$ in $\mathrm{T}$ cells, dendritic cell differentiation and natural killer (NK) cell biology [7-9]. Many ncRNAs were involved in the NF- $\mathrm{B}$, JAK-STAT and MAPK signaling [10] as well as regulated autoimmunity- and inflammation-related processes.

\section{miRNAs in rheumatic diseases}

miRNAs are small ncRNAs (18-25 nucleotides long), which regulate gene expression at the post-transcriptional level [11]. miRNAs inhibit translation and decrease mRNA stability by binding to the $3^{\prime} U T R$ of a target gene [12]. miRNAs affect nearly every phase of the cell cycle, from proliferation to differentiation to apoptosis, and play important roles in rheumatic diseases [13,14]. 


\section{miRNAs in SLE}

SLE is a chronic autoimmune disorder with heterogeneous clinical manifestations, which involve the skin, kidneys, joints, central nervous system (CNS), etc. $[15,16]$. The development of SLE involves nearly the entire immune system [17]. Altered miRNA expression profiles have been found in many types of immune cells in the context of SLE. In peripheral blood mononuclear cells (PBMCs), nine upregulated and seven downregulated miRNAs were found in SLE [18]. MiR-155, miR-17 and miR-181b were found to be significantly decreased in untreated patients, and miR-17 and miR-181b expression negatively correlated with the expression of the target genes activation-induced cytidine deaminase and IFN- $\alpha$ [19]. In CD4 ${ }^{+} \mathrm{T}$ cells, miR-29b, miR-21, miR-148a and miR-126 are upregulated and repress the expression of DNMT1, resulting in the overexpression of autoimmune-related genes [20-23]. The downregulation of miR-155 and miR-410 regulates the expression of IL-21 and IL-10, respectively [24,25]. MiR$142-3 \mathrm{p}$ is increased in monocyte-derived dendritic cells and promotes the secretion of SLE-related proinflammatory cytokines, such as CCL2, CCL5, CXCL8, IL-6 and TNF- $\alpha$ [26]. The downregulation of miR-146a in PBMCs and of miR-302d in monocytes negatively regulates the interferon pathway and contributes to the pathogenesis of SLE $[18,27]$.

Among serum miRNAs, miR-221-5p, miR-380-3p, miR-556-5p, miR-758-3p and miR-3074-3p are potential biomarkers of renal damage, and renal damage and disease activity are negatively correlated with miR-31 and positively correlated with miR-130b-3p [28,29]. In urinary exosomes, miR-29c might be a predictor of early renal fibrosis, and miR-146a can discriminate active lupus nephritis [30,31]. In MRL/MpOlaHsd-Tnfrsf6lp (MRL/lpr) mice, miR-410 regulates IL-6 and participates in renal fibrosis [32]. In the pristane-induced lupus model, miR-155deficient mice showed lower autoantibody levels and less severe nephritis [33].

\section{miRNAs in RA}

RA is a systemic autoimmune disease characterized by immune-mediated cartilage and subchondral bone damage that leads to joint deformities [34,35]. MiR-103a-3p is increased in whole blood cells and PBMCs in at-risk, autoantibody-positive asymptomatic first-degree relatives prior to RA onset and therefore may be a biomarker of preclinical RA [36]. MiR-155 and miR-146 are increased in synovial fibroblasts, synovial tissue and PBMCs in patients with RA [37-39]. MiR-146a promotes a proinflammatory Treg phenotype by increasing STAT1 activation, and miR-155 inhibits spontaneous monocyte apoptosis and influences B-cell function through PU.1 in RA patients [40-42]. MiR-29a and miR-27a are markedly downregulated in serum, synovial tissues and fibroblast-like synoviocytes (FLSs) of RA patients. MiR-29a inhibits proliferation, and miR-27a inhibits the migration and invasion of RA-FLSs through the STAT3 and TLR4/NF- $\mathrm{B}$ pathways, respectively [43,44]. MiR-125b is overexpressed in serum, synovial tissues and lipopolysaccharide (LPS)-stimulated FLSs and induces TNF- $\alpha$, IL-6, IL-1 $\beta$ and p65 expression to promote inflammation in RA [45].

Proinflammatory cytokines regulate the expression of multiple miRNAs in immune cells in RA. TNF- $\alpha$ promotes T-cell dysfunction by regulating miRNA expression in $\mathrm{T}$ cells. 13 miRNAs were altered in Jurkat cells after stimulation with TNF- $\alpha$, among which nine (miR-139-3p, miR-204, miR-760, miR-524-5p, miR-136, miR-548d3 p, miR-214, miR-383 and miR-887) are downregulated in T cells from RA patients. Moreover, the expression of four miRNAs (miR-139-3p, miR-204, miR-214 and miR-760) increased after treatment with biologic agents. These results suggest that TNF- $\alpha$ regulates miRNA expression in T cells and contributes to the immunopathogenesis of RA [46].

Among serum miRNAs, miR-155, miR-146a and miR-16 are dysregulated in early RA (ERA) and may be biomarkers of the early stage of this disease [47]. MiR-223 correlates with disease activity, and miR-16 and miR-223 are possible predictors of the outcome of ERA. MiR-125b is also a potential predictor of the response to rituximab treatment [48]. MiR-22, miR-486-3p and miR-382 are associated with the progression from systemic autoimmunity to RA inflammation [49]. MiR-125b was downregulated in PBMCs and plasma from treatment-naive ERA patients but was significantly upregulated after 3 months of treatment; thus, this miRNA may be a predictor of the curative effect [50].

\section{miRNAs in IIM}

IIMs are a group of heterogeneous disorders that cause damage to multiple organs and involve a common pathogenesis in which muscle weakness results from chronic inflammation of skeletal muscle [51]. The main subgroups of IIM are dermatomyositis (DM), polymyositis (PM), immune-mediated necrotic myopathy and inclusion body myositis. In PBMCs and serum from patients with DM, miR-206 is decreased and negatively 
correlates with the percentage of Th17 cells [52]. MiR-146a can regulate inflammatory macrophage infiltration in PM/DM by targeting TRAF6 and IL-17/ICAM-1 pathways [53]. In muscle tissue, miR-1, miR-133a and miR$133 \mathrm{~b}$ are decreased in all subtypes of IIM, and miR-206 is decreased in DM [54]. All these miRNAs play important roles in adult skeletal muscle differentiation, and their expression can be inhibited by TNF $\alpha$ [54]. Decreased miR-1 and miR-206 levels are negatively associated with muscle regeneration. Increased miR-155 and miR-146 levels are associated with autoimmunity [55]. In juvenile DM, 16 miRNAs are dysregulated in muscle tissue, with the greatest decrease observed in miR-10a [56]. The miRNA expression profiles of patients with DM and those with untreated juvenile dermatomyositis are different.

Regarding serum miRNAs, a previous study comprehensively compared the levels of circulating miRNAs in DM, PM and SLE patients and controls. Six miRNAs were differentially expressed in IIM patients compared with controls, three in DM and six in PM. Three miRNAs were related to disease activity. Moreover, 26 miRNAs were significantly differentially expressed in SLE patients compared with IIM patients [57]. These data suggest that miRNA profiles vary among different autoimmune diseases as well as among different subtypes of IIM. The plasma level of miR-4442 significantly decreased in patients with IIM after treatment and was significantly increased in patients with active PM/DM. MiR-4442 has been suggested to be a biomarker for monitoring disease activity and treatment response [58].

\section{miRNAs in SSc}

SSc is a complex, progressive autoimmune disease characterized by microvascular changes, extensive fibrosis in multiple organs and distinct autoantibodies. The main subgroups of SSc are limited cutaneous SSc and diffuse cutaneous SSc [59,60]. Specific miRNA expression signatures have been identified in lesion skin tissues of SSc. Many of the dysregulated miRNAs are involved in fibrotic processes [61,62]. Among the ten significantly downregulated miRNAs, five belong to the let-7 family. Inhibition of the most down-regulated let-7 member, let-7a, in fibroblasts induced the expression of type I collagen [63].

The miRNA expression signature was also evaluated in SSc fibroblasts. Among the dysregulated miRNAs, miR29a is strongly downregulated, inducing SSc fibroblast apoptosis and repressing the production of extracellular matrix, and type I and III collagen in fibroblasts [64,65]. The expression of miR-150 and miR-196a is decreased in SSc fibroblasts, contributing to the upregulation of type I collagen expression [66,67]. Both miR-4458 and miR-18a downregulate collagen expression via IL-23 and IL-23 in SSc fibroblasts [68]. In SSc hair samples, miR-196a and miR-29a are significantly decreased, and these miRNAs may be independent promising biomarkers of disease activity [69,70].

A total of 185 dysregulated miRNAs were identified in lung tissue from SSc patients with interstitial lung disease (SSc-ILD); among these miRNAs, miR-155 and miR-143 levels strongly correlate with the high-resolution computed tomography (HRCT) score. The expression of miR-155 in PBMCs significantly correlates with lung function in SSc-ILD [71]. Moreover, miR-155 is significantly upregulated in SSc lung fibroblasts via activation of the NLRP3 inflammasome [72].

Among serum miRNAs, miR-16, miR-223 and miR-638 are altered in SSc, while miR-142-3p, miR-150, miR-223 and miR-638 are dysregulated in SLE and SSc [73]. MiR-223, miR-181b, miR-342-3p and miR-184 are differentially expressed between limited cutaneous SSc and diffuse cutaneous SSc. Moreover, five specific miRNAs are associated with autoantibody profiles [74]. MiR-21, miR-92a and miR-483-5p are upregulated in SSc and are involved in skin fibrosis, inflammation and immune cell regulation [75-77]. MiR-30b is significantly downregulated in SSc, and this downregulation negatively correlates with the modified Rodnan skin score. In addition, miR-30b was confirmed to be upregulated in the skin of SSc patients and animal models [78].

\section{miRNAs in Pss}

pSS is an autoimmune disease caused by chronic inflammation in which the salivary and lacrimal glands are gradually damaged by infiltrating lymphocytes and plasma cells $[79,80]$. In the PBMCs of pSS patients, miRNA array analysis indicated that 25 miRNAs are overexpressed; among them, miR-146a, miR-16 and miR-21 are also increased in samples from patients with SLE. MiR-223 and miR-483-5p are upregulated. The upregulation of miR-146a and downregulation of miR-155 correlate with disease activity [81,82]. MiR-181a was found to be increased in Chinese patients [83]. Let-7d-3p, miR-155-5p, miR-222-3p, miR-30c-5p, miR-146a-5p, miR-378a$3 p$ and miR-28-5p are dysregulated in $\mathrm{CD}^{+} \mathrm{T}$ lymphocytes, and their potential target mRNAs are enriched in cancer and PI3K-Akt and TGF- $\beta$ signaling pathways. MiR-378a-3p, miR-222-3p, miR-26a-5p, miR-30b-5p 
and hsa-miR-19b-3p are dysregulated in B lymphocytes, and the potential target mRNAs are enriched in cancer, and the Wnt and PI3K-Akt signaling pathways [84]. MiR-146a-5p is increased in T cells but decreased in B cells, indicating different functions, such as promoting the release of inflammatory cytokines and inhibiting the expression of interferon-related genes [84]. In the salivary gland, miR-768-3p is increased, and miR-574 is decreased and correlates with clinical features [85]. The Ro/Sjögren's syndrome-related antigen A (Ro/SSA) and La/Sjögren's syndrome-related antigen B (La/SSB) autoantigens appear to be crucial in the immune response in pSS. Let7b, miR-16, miR-181a, miR-200b-3p, miR-200b-5p, miR-223 and miR-483-5p have been predicted to target Ro/SSA and La/SSB mRNAs. MiR-16 is upregulated in minor salivary gland tissues and positively correlated with Ro52/TRIM21 [86].

\section{IncRNAs in rheumatic diseases}

lncRNAs are RNA transcripts longer than 200 nucleotides with no coding potential that exert broad roles in cancer, viral infection, diabetes and other human diseases [87]. Recently, great advances have been made in understanding the function of lncRNAs in the immune system. Accumulating evidence suggests that lncRNAs play key roles in the pathogenesis of rheumatic diseases through RNA-DNA, RNA-RNA and RNA-protein interactions [88].

\section{IncRNAs in SLE}

In the PBMCs and monocytes of patients with SLE, MALAT1 and NEAT1 are increased, while linc0949 is decreased. MALAT1 is a nuclear lncRNA of approximately 8000 nucleotides and is located in nuclear speckles [89]. Silencing MALAT1 significantly reduces the expression of IL-21, and MALAT1 also regulates SIRT1 signaling [90]. NEAT1 is positively correlated with disease activity and induces the expression of proinflammatory chemokines, such as CCL2, CCL3, CCL4, CCL8, CXCL9, CXCL10 and CXCL11 [91]. These chemokines play critical roles in recruiting inflammatory cells into tissue. Lupus nephritis is characterized by the infiltration of Th1 cells in renal tissue, and Th1 cells overexpress chemokine receptors for NEAT1-regulated chemokines, including CXCR3, CCR5 and CCR2 [92-95]. NEAT1 might participate in the pathogenesis of tissue damage in SLE. Thus, NEAT1 could be a biomarker of early inflammation and organ damage in SLE patients. Linc0949 negatively correlates with the SLE disease activity index score, incidence of lupus nephritis and levels of complement C3 [96]. Interestingly, linc0949 expression is significantly increased in patients with a severe disease flare after treatment with immunosuppressive agents. The level of linc0949 did not change in palmitoyl-3-cysteine-serine-lysine-4-stimulated PBMCs from patients with SLE, which indicated an intrinsic immunodeficiency of SLE patients [96]. Therefore, linc0949 is a potential novel biomarker of SLE disease activity and treatment response. IncRNA GAS5 levels are significantly lower in B cells and CD4 ${ }^{+} \mathrm{T}$ cells of patients SLE than in those of controls, whereas no difference is seen in whole blood leukocytes [97]. There are six SNPs in the promoter region of GAS5 in BXSB mice, a mouse model of spontaneous lupus nephritis (LN), that have been discovered to strongly correlate with the 11-fold decrease in GAS5 expression [97]. GAS5 shows cell type-specific expression, and there is lower GAS5 expression in metabolically active tissue than in immune tissue. There are two glucocorticoid response element sequences in the GAS5 transcript, which can inhibit glucocorticoid-mediated gene transcription by competitive binding with the DNA-binding domain of the glucocorticoid receptor. Therefore, GAS5 specifically regulates the cellular metabolic status and promotes cell susceptibility to apoptosis. The lncRNA CYP2C91 [98] has been associated with regulator genes such as the transcription factor PU.1 (SPI), MSR1 and CCR1, which are all implicated in SLE pathogenesis. The lncRNAs XIST and its antisense gene TSIX are positively correlated with $\mathrm{X}$ inactivation, which may regulate $\mathrm{X}$ chromosome dosage in SLE morbidity [99].

\section{IncRNAs in RA}

HOTAIR is increased in PBMCs, bone marrow mononuclear cells and serum exosomes of patients with RA but decreased in synovial fibroblasts and osteoclasts $[100,101]$. HOTAIR interacts with histone-modifying protein complexes, induces the expression of histone-modified target genes and represses the expression of MMPs. HOTAIR upregulation in exosomes might induce macrophage accumulation in target tissues, and HOTAIR downregulation in synoviocytes and osteoclasts could induce the expression of MMP2 and MMP13, which are involved in the dissolution of cartilage matrix and bone, contributing to joint destruction. In the RA mouse model, HOTAIR overexpression can inhibit inflammation and promote cell proliferation by regulating the NF- $\mathrm{B}$ pathway, and this process can be reversed by miR-138 [102]. Given its stability and detectability, HOTAIR could be a biomarker of RA diagnosis and disease activity. Compared with healthy individuals or individuals with joint trauma, those 


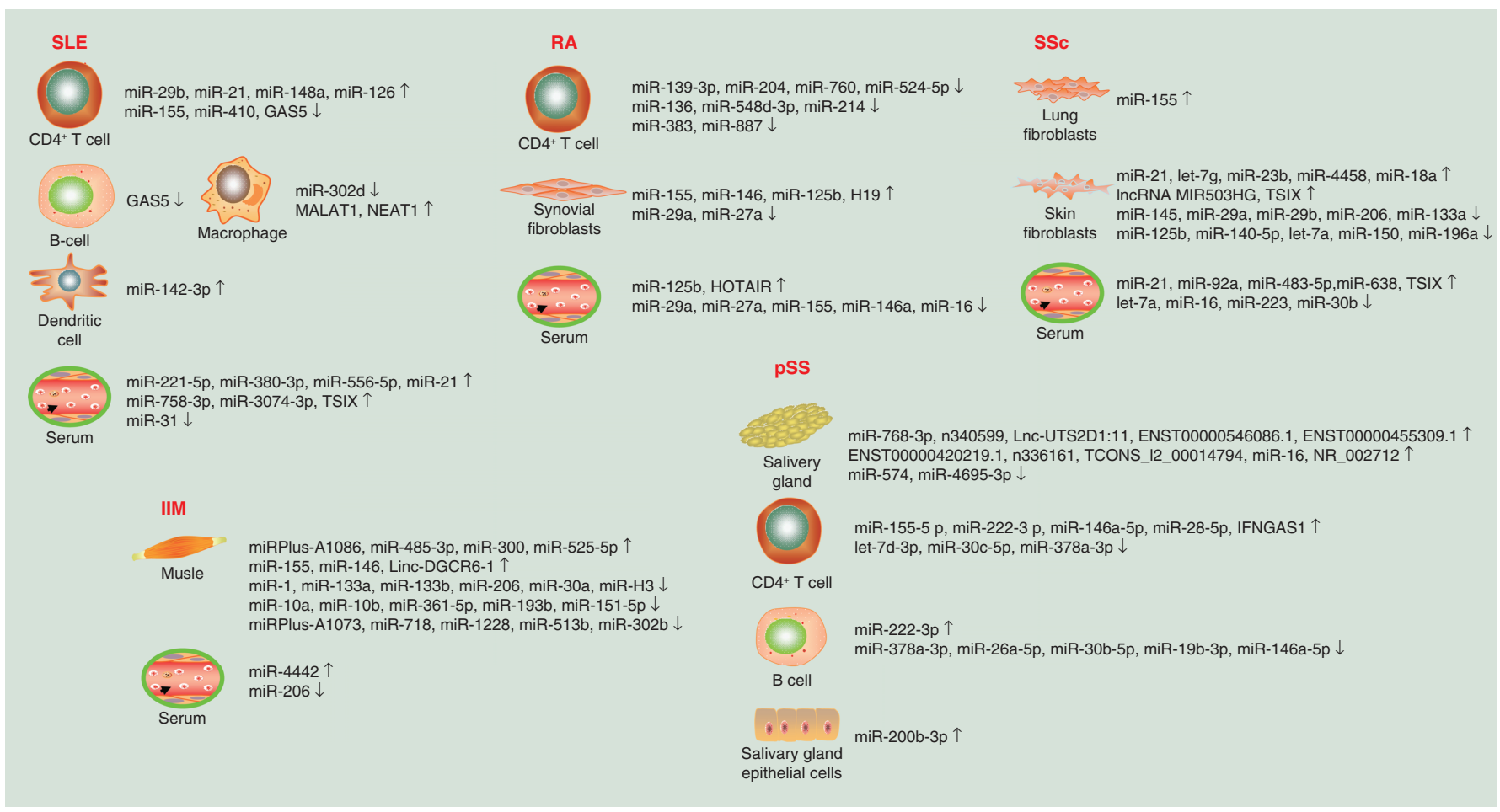

Figure 1. ncRNA dysregulation in rheumatic diseases.

IIM: Idiopathic inflammatory myopathy; pSS: Sjögren's syndrome; RA: Rheumatoid arthritis; SLE: Systemic lupus erythematous; SSc: Systemic sclerosis.

with RA and osteoarthritis (OA) have increased H19 expression in synovial tissue [103]. The expression of H19 significantly increased in synovial fibroblasts after serum starvation, and H19 expression is higher in RA than in OA $[103,104]$. Therefore, H19 might promote inflammation and joint damage in RA. The expression of the lncRNA TRP53COR1 is decreased in PBMCs from patients with RA and recovers after treatment with methotrexate [105]. Upon comparison of the IncRNA profiles in monocytes from RA patients treated with anti-IL-6R (tocilizumab) or anti-TNF (adalimumab), approximately 750 lncRNAs were found to be altered, suggesting that lncRNAs are highly specifically regulated by cytokines.

\section{IncRNAs in IIM}

Aberrant expression patterns of lncRNAs are found in DM muscle tissues. Differentially expressed lncRNAs and related pathways were also identified in DM patients with or without ILD or Jo-1 autoantibodies. Linc-DGCR6-1 is highly upregulated in the perifascicular areas of muscle fibres and might regulate the type 1 interferon-inducible gene USP18. The mRNA and protein levels of USP18 are increased identically to those of type 1 interferon-inducible genes [106].

\section{IncRNAs in SSC}

The IncRNA TSIX is increased in serum and skin fibroblasts of patients with SSc [107], which may be related to the TGF- $\beta$ signaling pathway $[108,109]$. The expression of type I collagen in SSc fibroblasts is reduced by TSIX siRNA and increased by overexpression of TSIX. TSIX expression is positively correlated with the modified Rodnan skin score. Therefore, TSIX may be a biomarker and therapeutic target for SSc. The IncRNA MIR503HG is expressed in SSc skin tissue, and TGF- $\beta$ can induce the expression of MIR503HG in fibroblasts and pulmonary vascular smooth muscle cells. The MIR503HG inhibitors SD208 and SB31532 can significantly reduce the expression of type I collagen, fibronectin and $\alpha$-smooth actin. MIR503HG may play an important role in the development of myofibroblasts in the context of SSc [110]. 
Table 1. The altered miRNAs in autoimmune diseases.

Sample miRNA

Level

Ref.

SLE

PBMCS miR-146a, miR-17, miR-181b

Downregulated

$[18,19]$

$\mathrm{CD}^{+} \mathrm{T}$ cells

miR-29b, miR-21, miR-148a, miR-126

Upregulated

$[20,23]$

miR-155, miR-410

Downregulated

$[24,25]$

Monocyte-derived DCs

miR-142-3p

Upregulated

[26]

Monocytes

miR-302d

Downregulated

[27]

Serum

miR-221-5p, miR-380-3p, miR-556-5p, miR-758-3p, miR-3074-3p, miR-21

Upregulated

$[28,29]$

Urinary exosomes

miR-31

Downregulated

miR-29c

Downregulated

miR-146a

Upregulated

\section{RA}

Whole blood cells miR-103a-3p

Upregulated

PBMCs

miR-103a-3p, miR-155, miR-146

Upregulated

[37-39]

$\operatorname{miR}-125 b$

T cells

miR-139-3p, miR-204, miR-760, miR-524-5p, miR-136, miR-548d-3p,

Downregulated

[50]

miR-214, miR-383, miR-887

Synovial fibroblasts and synovial miR-155, miR-146, miR-125b

Downregulated

tissue

Serum

miR-29a, miR-27a

Upregulated

miR-29a, miR-27a

Downregulated

miR-125

Upregulated

miR-29a, miR-27a, miR-155, miR-146a, miR-16

Downregulated

$[43,44,47]$

IIM

\begin{tabular}{|l|}
\hline PBMCs \\
\hline Muscle \\
\hline
\end{tabular}

miR-206

miR-1, miR-133a, miR-133b, miR-206

Downregulated

[53]

miR-155, miR-146

Muscle (JDM)

miR-10a, miR-10b, miR-30a, miR-361-5p, miR-193b, miR-151-5p, miRPlus-A1073, miR-718, miR-1228, miR-513b, miR-302b, miR-H3

miRPlus-A1086, miR-485-3p, miR-300, miR-525-5p

Downregulated

Upregulated

Downregulated

Upregulated

Serum

miR-206

Upregulated

Downregulated

SSc

Skin tissues and fibroblasts

miR-21, let-7 g, miR-23b

Upregulated

miR-145, miR-29b, miR-206, miR-133a, miR-125b, miR-140-5p, let-7a

Downregulated

Skin fibroblasts

miR-21, miR-4458, miR-18a

miR-145, miR-29b, miR-29a, miR-150, miR-196a

Upregulated

$[61,64-67]$

Lung fibroblasts

miR-155

Downregulated

[72]

Hair

miR-196a, miR-29a

Upregulated

$[69,70]$

Serum

miR-21, miR-92a, miR-483-5p,miR-638

Downregulated

let-7a, miR-16, miR-223, miR-30b

$[63,73,78]$

pSS

Downregulated

[81-83]

PBMC

miR-146a, miR-16, miR-21, miR-223, miR-483-5p, miR-181a

Upregulated

[82]

$\mathrm{CD}^{+}{ }^{+} \mathrm{T}$ cells

miR-155-5p, miR-222-3p, miR-146a-5p, miR-28-5p

Downregulated

let-7d-3p, miR-30c-5p, miR-378a-3p

Upregulated

B cells

miR-222-3p

Downregulated

miR-378a-3p, miR-26a-5p, miR-30b-5p, miR-19b-3p, miR-146a-5p

Upregulated

Salivary gland

miR-768-3p, miR-16

Downregulated

$[85,86]$

miR-574, miR-4695-3p

Upregulated

$[85,86]$

Salivary gland epithelial cells

miR-200b-3p

Upregulated

[86]

DC: Dendritic cell; IIM: Idiopathic inflammatory myopathy; JDM: Juvenile dermatomyositis; PBMC: Peripheral blood mononuclear cell; pSS: Sjögren's syndrome; RA: Rheumatoid arthritis; SLE: Systemic lupus erythematosus; SSc: Systemic sclerosis. 
Table 2. The altered IncRNAs in autoimmune disease.

\begin{tabular}{|c|c|c|c|c|}
\hline IncRNA & Tissue/cell & Level & Function & Ref. \\
\hline \multicolumn{5}{|l|}{ SLE } \\
\hline MALAT1 & PBMCs; monocytes & Upregulated & $\begin{array}{l}\text { Negatively related to the level of SIRT1; positive } \\
\text { correlated to the level of IL- } 21\end{array}$ & {$[89,90]$} \\
\hline Linc0949 & PBMCs & Downregulated & $\begin{array}{l}\text { Organ involvement; negative correlation with } \\
\text { SLEDAI score; the level of complement protein C3 }\end{array}$ & [96] \\
\hline NEAT1 & PBMCs; monocytes & Upregulated & Increases secretion of cytokines such as IL-6 & [91-95] \\
\hline GAS5 & $\begin{array}{l}\text { B cells } \\
\mathrm{CD}^{+} \mathrm{T} \text { cells }\end{array}$ & Downregulated & $\begin{array}{l}\text { Cell-type-specific expression; correlated with } \\
\text { metabolism in immune tissue }\end{array}$ & [97] \\
\hline IncRNA CYP2C9 & Nucleus & Upregulated & Association with SLE pathogenesis fragments & [98] \\
\hline TSIX & Serum & Upregulated & $\begin{array}{l}\text { Correlated with } \mathrm{X} \text { chromosome dosage in SLE } \\
\text { morbidity }\end{array}$ & [99] \\
\hline \multicolumn{5}{|l|}{ RA } \\
\hline H19 & $\begin{array}{l}\text { Inflamed synovial tissue; synovial } \\
\text { fibroblasts }\end{array}$ & Upregulated & $\begin{array}{l}\text { Has strong correlation with the expression of } \\
\text { imprinted gene network; contributes to } \\
\text { inflammatory status }\end{array}$ & {$[103,104]$} \\
\hline HOTAIR & $\begin{array}{l}\text { PBMCs; bone mononuclear cells; } \\
\text { serum exosomes }\end{array}$ & Upregulated & $\begin{array}{l}\text { Recruites polycomb repressive complex } 2 \text { to target } \\
\text { transcripts; contributes to joint destruction }\end{array}$ & {$[100-102]$} \\
\hline InCRNA TRP53COR1 & PBMCs & Downregulated & Specifically regulated by cytokines & [105] \\
\hline \multicolumn{5}{|l|}{ SSC } \\
\hline TSIX & Serum; skin & Upregulated & Stabilizes type I collagen mRNA & [107-109] \\
\hline IncRNA MIR503HG & Skin & Upregulated & Promote the development of myofibroblast & [110] \\
\hline \multicolumn{5}{|l|}{ pSS } \\
\hline $\begin{array}{l}\text { ENST00000455309.1, } \\
\text { ENST00000420219.1, NR_002712, } \\
\text { Lnc-UTS2D1:1, } \\
\text { ENST00000546086.1, n340599, } \\
\text { TCONS_I2_00014794 and n336161 }\end{array}$ & Labial salivary glands & Upregulated & $\begin{array}{l}\text { Have correlations with IgA, IgM, ESR, visual } \\
\text { analog scale of dry eyes and parotid swelling; } \\
\text { involve in chemokine signaling pathways }\end{array}$ & [111] \\
\hline IFNGAS1 & $\mathrm{CD}^{+}{ }^{+} \mathrm{T}$ cells & Upregulated & $\begin{array}{l}\text { Correlated with anti-SSA antibody, IgG level and } \\
\text { ESR }\end{array}$ & [112] \\
\hline \multicolumn{5}{|l|}{ IIM } \\
\hline Linc-DGCR6-1 & Muscle fibres & Upregulated & Regulate USP18 & [106] \\
\hline
\end{tabular}

IncRNAs in pSS

In pSS, eight IncRNAs are correlated with IgA and IgM levels, the erythrocyte sedimentation rate (ESR), and the visual analog scale (VAS) of dry eyes and parotid swelling; these lncRNAs are ENST00000455309.1, ENST00000420219.1, NR_002712, Lnc-UTS2D1:1, ENST00000546086.1, n340599, TCONS_12_00014794 and n336161 [111]. Two are associated with the VAS of parotid swelling, two with rheumatoid factor, three with ESR, two with IgA, six with $\beta 2$ microglobulin, one with the VAS of dry eyes and six with disease course; moreover, four were significantly upregulated in SSB-positive patients compared with SSB-negative patients. However, the specific connection between these lncRNAs and disease has not been elucidated. IFNG antisense RNA (IFNGAS) levels are increased in $\mathrm{CD}^{+}{ }^{+} \mathrm{T}$ cells and positively correlate with anti-SSA antibody and serum IgG levels and the ESR. IFNG-AS1 can be regulated by T-bet, and the mRNA levels of both TBX21 (encoding T-bet) and IFNG are upregulated in $\mathrm{CD}^{+} \mathrm{T}$ cells [112]. The role of IFNG-AS in pSS is not fully understood and requires further investigation.

\section{circRNAs in rheumatic diseases}

circRNAs are a set of ncRNAs without $5^{\prime}$ and $3^{\prime}$ ends, characterized with closed loop structures [113]. circRNAs were first described in viroids in 1970s [114]. Due to its stability in mammalian cells, circRNAs were supposed to be a more ideal biomarker compared with lncRNAs and miRNAs [115,116]. The 'sponging' function of circRNAs reveals its regulatory mechanism that it can inhibit miRNA by targeting seed sequences. The regulatory potency of circRNAs has been well studied in several fields [117-119], only a few reports in rheumatic diseases. Here, we summarize the regulatory role of circRNAs in SLE and RA. 
circRNAs in SLE

Seven circRNAs (circHLA-C, circZNF609, circEKL4, circFAM188A, circUBR5, circPDE4B and circSP100) were found increased in renal samples of LN class IV patients compared with healthy controls [120]. Circular human leukocyte antigen-C (CircHLA-C) positively correlated with serum creatinine and LN disease activity, and negatively correlated with miR-150, which suggested circHLA-C might sponge miR-150 in the pathogenesis of LN. Plasma circRNA_002453 is increased in SLE patients compared with RA patients and healthy controls, and even higher in LN patients. The level of circRNA_002453 was associated with 24-h proteinuria and LN severity, but was not correlated with SLE disease activity index 2000 (SLEDAI-2 K) score, complement 3(C3) and complement 4(C4) [121]. Hsa_circ_0045272 was decreased in SLE peripheral T cells. Decreased hsa_circ_0045272 accelerated Jurkat cells apoptosis and promoted IL-2 secretion by down-regulating two mRNAs as its ceRNAs. However, no significant correlations were found between the levels of hsa_circ_0045272 and clinical index [122].

\section{circRNAs in RA}

Five circRNAs (104871, 092516, 003524, 103047, 101873) were increased in PBMCs of RA. circRNA_104871 was correlated with RA diagnosis, which might be an important biomarker for RA diagnosis [123]. High levels of hsa_circ_0044235 [124] and hsa_circ_0001859 [125] were found in RA patients. Hsa_circ_0001859 was involved in the chronic inflammatory of synovial tissue through regulating ATF2 level sponging miRNA-204/211.

\section{piRNAs in RA}

piRNAs are a class of small ncRNAs associated with Piwi proteins of Argonaute family, 24-31 nucleotides in length [126]. Up to 300 piRNAs were detected to be highly expressed in synovial tissues and synovial fibroblasts from RA and OA patients to some extent, of note, piR-16735 took 20\% account, piR-18570, -17724 and -20388 each covered $5 \%$ of all piRNA reads, but were probably not involved in the regulation of RA synovial fibroblast proliferation [127] (Figure 1 and Tables $1 \& 2$ ).

\section{Conclusion \& future perspective}

Rheumatic diseases are a group of complex diseases involving pathophysiological changes in multiple organs. Advances in analyzing pathogenesis in recent years have improved our understanding of these diseases. ncRNAs have emerged as important epigenetic modifiers that participate in rheumatic diseases and exert critical roles. However, no reviews had completely summarized the role of ncRNAs in rheumatic diseases. In this review, for the first time, we summarize the function of ncRNAs, especially lncRNAs and miRNAs, in the pathogenesis of rheumatic diseases and discuss potential biomarkers of diagnosis, treatment and prognosis. A variety of ncRNAs were observed to be differentially expressed in rheumatic diseases, whereas, only a few of them were identified as specific roles in the process of diseases. ncRNAs are ideal noninvasive biomarkers due to its stability, and even more likely to be predominant source of biomarker in the following decades. Much more developed technologies will be applied to supply a gap of their interaction partners and network mechanism. More basic studies are needed in the future to explore the functional role of various ncRNAs in immune cells and specific tissues to clarify ncRNA-based immune mechanisms. Given the important role in pathogenesis of rheumatic diseases, the exploitation of ncRNAs may provide effective diagnostic approaches and novel ncRNAs-targeting therapeutic strategies for rheumatic diseases into clinical use and could depict a more specific blueprint of molecular biomarkers.

\section{Executive summary}

- miRNAs destroy the stability of RNA and inhibit translation process via binding to the $3^{\prime}$ UTR of target gene. miRNAs also serve as biomarkers for rheumatic diseases, and potential therapeutic targets.

- IncRNAs expression is tissue and disease specific at different rheumatic disease stages, involved in disease pathogenesis by RNA-DNA, RNA-RNA and RNA-protein interaction.

- The role of piRNAs and circRNAs in the pathogenesis of autoimmune diseases remains ill defined. Whether the sponging function of circRNAs is involved in regulating RNA or protein expression in rheumatic diseases is still elusive.

- ncRNAs are involved in multiple aspects of rheumatic diseases, including gene transcription, immune cells differentiation and apoptosis, immune responses, disease activity and so forth. 
Author contributions

W Chen and D Liu wrote the first draft. Q-Z Li revised the manuscript. H Zhu has revised the final version and added extra information.

Financial \& competing interests disclosure

This study was funded by grants from Hunan Provincial Natural Science Foundation (2018JJ3823), National Natural Science Foundation of China (81671621) and Fundamental Research Funds for the Central Universities of Central South University (2018zzts247). The authors have no other relevant affiliations or financial involvement with any organization or entity with a financial interest in or financial conflict with the subject matter or materials discussed in the manuscript apart from those disclosed.

No writing assistance was utilized in the production of this manuscript.

\section{Open access}

This work is licensed under the Attribution-NonCommercial-NoDerivatives 4.0 Unported License. To view a copy of this license, visit http://creativecommons.org/licenses/by-nc-nd/4.0/Openaccess This work is licensed under the Attribution-NonCommercialNoDerivatives 4.0 Unported License. To view a copy of this license, visit http://creativecommons.org/licenses/by-nc-nd/4.0/

\section{References}

Papers of special note have been highlighted as: $\bullet$ of interest; $\bullet \bullet$ of considerable interest

1. Coit P, Ognenovski M, Gensterblum E, Maksimowicz-Mckinnon K, Wren JD, Sawalha AH. Ethnicity-specific epigenetic variation in naive CD4 ${ }^{+}$T cells and the susceptibility to autoimmunity. Epigenetics Chromatin 8, 49 (2015).

2. Long H, Wang X, Chen Y, Wang L, Zhao M, Lu Q. Dysregulation of microRNAs in autoimmune diseases: pathogenesis, biomarkers and potential therapeutic targets. Cancer Lett. 428, 90-103 (2018).

-. Comprehensive review of miRNA dysregulation implicated in autoimmune diseases.

3. Kolarz B, Majdan M. Epigenetic aspects of rheumatoid arthritis: contribution of non-coding RNAs. Semin. Arthritis Rheum. 46(6), 724-731 (2017).

4. Lodish HF, Zhou B, Liu G, Chen CZ. Micromanagement of the immune system by microRNAs. Nat. Rev. Immunol. 8(2), 120-130 (2008).

5. Neilson JR, Zheng GX, Burge CB, Sharp PA. Dynamic regulation of miRNA expression in ordered stages of cellular development. Genes Dev. 21(5), 578-589 (2007).

6. Lai NS, Koo M, Yu CL, Lu MC. Immunopathogenesis of systemic lupus erythematosus and rheumatoid arthritis: the role of aberrant expression of non-coding RNAs in T cells. Clin. Exp. Immunol. 187(3), 327-336 (2017).

7. Yang C, Shen C, Feng T, Li H. Noncoding RNA in NK cells. J. Leukoc. Biol. 105 (1), 63-71 (2018).

8. Gomez JA, Wapinski OL, Yang YW et al. The NeST long ncRNA controls microbial susceptibility and epigenetic activation of the interferon- $\gamma$ locus. Cell 152(4), 743-754 (2013).

9. Wang P, Xue Y, Han Y et al. The STAT3-binding long noncoding RNA lnc-DC controls human dendritic cell differentiation. Science 344(6181), 310-313 (2014).

10. Chew CL, Conos SA, Unal B, Tergaonkar V. Noncoding RNAs: master regulators of inflammatory signaling. Trends Mol. Med. 24(1), 66-84 (2018).

11. Chen CZ, Li L, Lodish HF, Bartel DP. MicroRNAs modulate hematopoietic lineage differentiation. Science 303(5654), 83-86 (2004).

12. Wu L, Belasco JG. Let me count the ways: mechanisms of gene regulation by miRNAs and siRNAs. Mol. Cell 29(1), 1-7 (2008).

13. Baltimore D, Boldin MP, O’Connell RM, Rao DS, Taganov KD. MicroRNAs: new regulators of immune cell development and function. Nat. Immunol. 9(8), 839-845 (2008).

14. O’Connell RM, Rao DS, Chaudhuri AA, Baltimore D. Physiological and pathological roles for microRNAs in the immune system. Nat. Rev. Immunol. 10(2), 111-122 (2010).

15. Systemic lupus erythematosus. Nat. Rev. Dis. Primers 2, 16040 (2016).

16. Kaul A, Gordon C, Crow MK et al. Systemic lupus erythematosus. Nat. Rev. Dis. Primers 2, 16039 (2016).

17. Lang KS, Burow A, Kurrer M, Lang PA, Recher M. The role of the innate immune response in autoimmune disease. $J$. Autoimmun. 29(4), 206-212 (2007).

18. Smith S, Fernando T, Wu PW et al. MicroRNA-302d targets IRF9 to regulate the IFN-induced gene expression in SLE. J. Autoimmun. 79, 105-111 (2017).

19. Kaga H, Komatsuda A, Omokawa A et al. Downregulated expression of miR-155, miR-17, and miR-181b, and upregulated expression of activation-induced cytidine deaminase and interferon-alpha in PBMCs from patients with SLE. Mod. Rheumatol. 25(6), 865-870 (2015). 
20. Pan W, Zhu S, Yuan M et al. MicroRNA-21 and microRNA-148a contribute to DNA hypomethylation in lupus $\mathrm{CD}^{+}{ }^{+} \mathrm{T}$ cells by directly and indirectly targeting DNA methyltransferase 1. J. Immunol. 184(12), 6773-6781 (2010).

21. Zhao S, Wang Y, Liang Y et al. MicroRNA-126 regulates DNA methylation in $\mathrm{CD}^{+} \mathrm{T}$ cells and contributes to systemic lupus erythematosus by targeting DNA methyltransferase 1. Arthritis Rheum. 63(5), 1376-1386 (2011).

22. Wang Z, Lu Q, Wang Z. Epigenetic alterations in cellular immunity: new insights into autoimmune diseases. Cell Physiol. Biochem. 41(2), 645-660 (2017).

23. Qin $\mathrm{H}$, Zhu X, Liang J et al. MicroRNA-29b contributes to DNA hypomethylation of $\mathrm{CD} 4^{+} \mathrm{T}$ cells in systemic lupus erythematosus by indirectly targeting DNA methyltransferase 1. J. Dermatol. Sci. 69(1), 61-67 (2013).

24. Rasmussen TK, Andersen T, Bak RO et al. Overexpression of microRNA-155 increases IL-21 mediated STAT3 signaling and IL-21 production in systemic lupus erythematosus. Arthritis Res. Ther. 17, 154 (2015).

25. Liu D, Zhang N, Zhang X, Qin M, Dong Y, Jin L. MiR-410 down-regulates the expression of interleukin-10 by targeting STAT3 in the pathogenesis of systemic lupus erythematosus. Cell Physiol. Biochem. 39(1), 303-315 (2016).

26. Wang Y, Liang J, Qin H et al. Elevated expression of miR-142-3p is related to the pro-inflammatory function of monocyte-derived dendritic cells in SLE. Arthritis Res. Ther. 18(1), 263 (2016).

27. Tang $\mathrm{Y}$, Luo X, Cui H et al. MicroRNA-146A contributes to abnormal activation of the type I interferon pathway in human lupus by targeting the key signaling proteins. Arthritis Rheum. 60(4), 1065-1075 (2009).

28. Navarro-Quiroz E, Pacheco-Lugo L, Lorenzi H et al. High-throughput sequencing reveals circulating miRNAs as potential biomarkers of kidney damage in patients with systemic lupus erythematosus. PLoS ONE 11(11), e0166202 (2016).

29. Amr KS, Bayoumi FS, Elgengehy FT, Abdallah SO, Ahmed HH, Eissa E. The role of microRNA-31 and microRNA-21 as regulatory biomarkers in the activation of T lymphocytes of Egyptian lupus patients. Rheumatol. Int. 36(11), 1617-1625 (2016).

30. Sole C, Cortes-Hernandez J, Felip ML, Vidal M, Ordi-Ros J. miR-29c in urinary exosomes as predictor of early renal fibrosis in lupus nephritis. Nephrol. Dial. Transplant. 30(9), 1488-1496 (2015).

31. Perez-Hernandez J, Forner MJ, Pinto C, Chaves FJ, Cortes R, Redon J. Increased urinary exosomal microRNAs in patients with systemic lupus erythematosus. PLoS ONE 10(9), e0138618 (2015).

32. Liu D, Zhang $\mathrm{N}$, Zhang J, Zhao H, Wang X. miR-410 suppresses the expression of interleukin- 6 as well as renal fibrosis in the pathogenesis of lupus nephritis. Clin. Exp. Pharmacol. Physiol. 43(6), 616-625 (2016).

33. Leiss H, Salzberger W, Jacobs B et al. MicroRNA 155-deficiency leads to decreased autoantibody levels and reduced severity of nephritis and pneumonitis in pristane-induced lupus. PLoS ONE 12(7), e0181015 (2017).

34. Smolen JS, Aletaha D, Barton A et al. Rheumatoid arthritis. Nat. Rev. Dis. Primers 4, 18001 (2018).

35. McInnes IB, Schett G. The pathogenesis of rheumatoid arthritis. N. Engl. J. Med. 365(323), 2205-2219 (2011)

36. Anaparti V, Smolik I, Meng X, Spicer V, Mookherjee N, El-Gabalawy H. Whole blood microRNA expression pattern differentiates patients with rheumatoid arthritis, their seropositive first-degree relatives, and healthy unrelated control subjects. Arthritis Res. Ther. 19(1), 249 (2017).

37. Nakasa T, Miyaki S, Okubo A et al. Expression of microRNA-146 in rheumatoid arthritis synovial tissue. Arthritis Rheum. 58(5), 1284-1292 (2008).

38. Stanczyk J, Pedrioli DM, Brentano F et al. Altered expression of MicroRNA in synovial fibroblasts and synovial tissue in rheumatoid arthritis. Arthritis Rheum. 58(4), 1001-1009 (2008).

39. Kurowska-Stolarska M, Alivernini S, Ballantine LE et al. MicroRNA-155 as a proinflammatory regulator in clinical and experimental arthritis. Proc. Natl Acad. Sci. USA 108(27), 11193-11198 (2011).

40. Zhou Q, Haupt S, Kreuzer JT et al. Decreased expression of miR-146a and miR-155 contributes to an abnormal Treg phenotype in patients with rheumatoid arthritis. Ann. Rheum. Dis. 74(6), 1265-1274 (2015).

41. Rajasekhar M, Olsson AM, Steel KJ et al. MicroRNA-155 contributes to enhanced resistance to apoptosis in monocytes from patients with rheumatoid arthritis. J. Autoimmun. 79, 53-62 (2017).

42. Alivernini S, Kurowska-Stolarska M, Tolusso B et al. MicroRNA-155 influences B-cell function through PU.1 in rheumatoid arthritis. Nat. Commun. 7, 12970 (2016).

43. Peng JS, Chen SY, Wu CL et al. Amelioration of experimental autoimmune arthritis through targeting of synovial fibroblasts by intraarticular delivery of microRNAs 140-3p and 140-5p. Arthritis Rheumatol. 68(2), 370-381 (2016).

44. Shi DL, Shi GR, Xie J, Du XZ, Yang H. MicroRNA-27a inhibits cell migration and invasion of fibroblast-like synoviocytes by targeting follistatin-like protein 1 in rheumatoid arthritis. Mol. Cells 39(8), 611-618 (2016).

45. Zhang B, Wang LS, Zhou YH. Elevated microRNA-125b promotes inflammation in rheumatoid arthritis by activation of NF- $\mathrm{kB}$ pathway. Biomed. Pharmacother. 93, 1151-1157 (2017).

46. Lai NS, Yu HC, Tung CH, Huang KY, Huang HB, Lu MC. The role of aberrant expression of $\mathrm{T}$ cell miRNAs affected by TNF- $\alpha$ in the immunopathogenesis of rheumatoid arthritis. Arthritis Res. Ther. 19(1), 261 (2017). 
47. Filkova M, Aradi B, Senolt L et al. Association of circulating miR-223 and miR-16 with disease activity in patients with early rheumatoid arthritis. Ann. Rheum. Dis. 73(10), 1898-1904 (2014).

48. Duroux-Richard I, Pers YM, Fabre $S$ et al. Circulating miRNA-125b is a potential biomarker predicting response to rituximab in rheumatoid arthritis. Mediators Inflamm. 2014, 342524 (2014).

49. Ouboussad L, Hunt L, Hensor EMA et al. Profiling microRNAs in individuals at risk of progression to rheumatoid arthritis. Arthritis Res. Ther. 19(1), 288 (2017).

50. Hruskova V, Jandova R, Vernerova L et al. MicroRNA-125b: association with disease activity and the treatment response of patients with early rheumatoid arthritis. Arthritis Res. Ther. 18(1), 124 (2016).

51. Lundberg IE, De Visser M, Werth VP. Classification of myositis. Nat. Rev. Rheumatol. 14(5), 269-278 (2018).

52. Tang X, Tian X, Zhang Y et al. Correlation between the frequency of Th17 cell and the expression of microRNA-206 in patients with dermatomyositis. Clin. Dev. Immunol. 2013, 345347 (2013).

53. Yin Y, Li F, Shi J, Li S, Cai J, Jiang Y. MiR-146a regulates inflammatory infiltration by macrophages in polymyositis/dermatomyositis by targeting TRAF6 and affecting IL-17/ICAM-1 pathway. Cell Physiol. Biochem. 40(3-4), 486-498 (2016).

54. Georgantas RW, Streicher K, Greenberg SA et al. Inhibition of myogenic microRNAs 1, 133, and 206 by inflammatory cytokines links inflammation and muscle degeneration in adult inflammatory myopathies. Arthritis Rheumatol. 66(4), 1022-1033 (2014).

55. Parkes JE, Day PJ, Chinoy H, Lamb JA. The role of microRNAs in the idiopathic inflammatory myopathies. Curr. Opin Rheumatol. 27(6), 608-615 (2015).

56. Xu D, Huang CC, Kachaochana A et al. MicroRNA-10a regulation of proinflammatory mediators: an important component of untreated juvenile dermatomyositis. J. Rheumatol. 43(1), 161-168 (2016).

57. Misunova M, Salinas-Riester G, Luthin S et al. Microarray analysis of circulating micro RNAs in the serum of patients with polymyositis and dermatomyositis reveals a distinct disease expression profile and is associated with disease activity. Clin. Exp. Rheumatol. 34(1), 17-24 (2016).

58. Hirai $\mathrm{T}$, Ikeda $\mathrm{K}$, Tsushima $\mathrm{H}$ et al. Circulating plasma microRNA profiling in patients with polymyositis/dermatomyositis before and after treatment: miRNA may be associated with polymyositis/dermatomyositis. Inflamm. Regen. 38, 1 (2018).

59. Allanore Y, Simms R, Distler O et al. Systemic sclerosis. Nat. Rev. Dis. Primers 1, 15002 (2015).

60. Denton CP, Khanna D. Systemic sclerosis. Lancet 390(10103), 1685-1699 (2017).

61. Zhu H, Li Y, Qu S et al. MicroRNA expression abnormalities in limited cutaneous scleroderma and diffuse cutaneous scleroderma. J. Clin. Immunol. 32(3), 514-522 (2012).

62. Li H, Yang R, Fan X et al. MicroRNA array analysis of microRNAs related to systemic scleroderma. Rheumatol. Int. 32(2), 307-313 (2012).

63. Makino K, Jinnin M, Hirano A et al. The downregulation of microRNA let-7a contributes to the excessive expression of type I collagen in systemic and localized scleroderma. J. Immunol. 190(8), 3905-3915 (2013).

64. Maurer B, Stanczyk J, Jungel A et al. MicroRNA-29, a key regulator of collagen expression in systemic sclerosis. Arthritis Rheum. 62(6), $1733-1743$ (2010).

65. Jafarinejad-Farsangi S, Farazmand A, Mahmoudi M et al. MicroRNA-29a induces apoptosis via increasing the Bax:Bcl-2 ratio in dermal fibroblasts of patients with systemic sclerosis. Autoimmunity 48(6), 369-378 (2015).

66. Honda N, Jinnin M, Kira-Etoh T et al. miR-150 down-regulation contributes to the constitutive type I collagen overexpression in scleroderma dermal fibroblasts via the induction of integrin $\beta 3$. Am. J. Pathol. 182(1), 206-216 (2013).

67. Honda N, Jinnin M, Kajihara I et al. TGF- $\beta$-mediated downregulation of microRNA-196a contributes to the constitutive upregulated type I collagen expression in scleroderma dermal fibroblasts. J. Immunol. 188(7), 3323-3331 (2012).

68. Nakayama W, Jinnin M, Tomizawa $Y$ et al. Dysregulated interleukin-23 signalling contributes to the increased collagen production in scleroderma fibroblasts via balancing microRNA expression. Rheumatology (Oxford) 56(1), 145-155 (2017).

69. Wang Z, Jinnin M, Kudo H et al. Detection of hair-microRNAs as the novel potent biomarker: evaluation of the usefulness for the diagnosis of scleroderma. J. Dermatol. Sci. 72(2), 134-141 (2013).

70. Takemoto R, Jinnin M, Wang Z et al. Hair miR-29a levels are decreased in patients with scleroderma. Exp. Dermatol. 22(12), 832-833 (2013).

71. Christmann RB, Wooten A, Sampaio-Barros P et al. miR-155 in the progression of lung fibrosis in systemic sclerosis. Arthritis Res. Ther. 18(1), 155 (2016).

72. Artlett CM, Sassi-Gaha S, Hope JL, Feghali-Bostwick CA, Katsikis PD. Mir-155 is overexpressed in systemic sclerosis fibroblasts and is required for NLRP3 inflammasome-mediated collagen synthesis during fibrosis. Arthritis Res. Ther. 19(1), 144 (2017).

-. First demonstration of the relation between Mir-155 and NLRP3 inflammasome in systemic sclerosis fibroblasts.

73. Steen SO, Iversen LV, Carlsen AL et al. The circulating cell-free microRNA profile in systemic sclerosis is distinct from both healthy controls and systemic lupus erythematosus. J. Rheumatol. 42(2), 214-221 (2015). 
74. Wuttge DM, Carlsen AL, Teku G et al. Specific autoantibody profiles and disease subgroups correlate with circulating micro-RNA in systemic sclerosis. Rheumatology (Oxford) 54(11), 2100-2107 (2015).

75. Sing T, Jinnin M, Yamane K et al. microRNA-92a expression in the sera and dermal fibroblasts increases in patients with scleroderma. Rheumatology (Oxford) 51(9), 1550-1556 (2012).

76. Zhu H, Luo H, Li Y et al. MicroRNA-21 in scleroderma fibrosis and its function in TGF- $\beta$-regulated fibrosis-related genes expression. $J$. Clin. Immunol. 33(6), 1100-1109 (2013).

77. Chouri E, Servaas NH, Bekker CPJ et al. Serum microRNA screening and functional studies reveal miR-483-5p as a potential driver of fibrosis in systemic sclerosis. J. Autoimmun. 89, 162-170 (2018).

78. Tanaka S, Suto A, Ikeda K et al. Alteration of circulating miRNAs in SSc: miR-30b regulates the expression of PDGF receptor $\beta$. Rheumatology (Oxford) 52(11), 1963-1972 (2013).

79. Brito-Zeron P, Baldini C, Bootsma H et al. Sjogren syndrome. Nat. Rev. Dis. Primers 2, 16047 (2016).

80. Singh N, Cohen PL. The T cell in Sjogren's syndrome: force majeure, not spectateur. J. Autoimmun. 39(3), 229-233 (2012).

81. Pauley KM, Stewart CM, Gauna AE et al. Altered miR-146a expression in Sjogren's syndrome and its functional role in innate immunity. Eur. J. Immunol. 41(7), 2029-2039 (2011).

82. Shi H, Zheng LY, Zhang P, Yu CQ. miR-146a and miR-155 expression in PBMCs from patients with Sjogren's syndrome. J. Oral. Pathol. Med. 43(10), 792-797 (2014).

83. Peng L, Ma W, Yi F et al. MicroRNA profiling in Chinese patients with primary Sjogren syndrome reveals elevated miRNA-181a in peripheral blood mononuclear cells. J. Rheumatol. 41(11), 2208-2213 (2014).

84. Wang-Renault SF, Boudaoud S, Nocturne G et al. Deregulation of microRNA expression in purified T and B lymphocytes from patients with primary Sjogren's syndrome. Ann. Rheum. Dis. 77(1), 133-140 (2018).

85. Alevizos I, Alexander S, Turner RJ, Illei GG. MicroRNA expression profiles as biomarkers of minor salivary gland inflammation and dysfunction in Sjogren's syndrome. Arthritis Rheum. 63(2), 535-544 (2011).

86. Gourzi VC, Kapsogeorgou EK, Kyriakidis NC, Tzioufas AG. Study of microRNAs (miRNAs) that are predicted to target the autoantigens Ro/SSA and La/SSB in primary Sjogren's syndrome. Clin. Exp. Immunol. 182(1), 14-22 (2015).

87. Quinn JJ, Chang HY. Unique features of long non-coding RNA biogenesis and function. Nat. Rev. Genet. 17(1), 47-62 (2016).

88. Mercer Tr DM, Mattick JS. Long non-coding RNAs: insights into functions. Nat. Rev. Genet. 10(3), 155-159 (2009).

- Comprehensive review of the rapidly advancing field of IncRNAs, describing their conservation, their organization in the genome and their roles in gene regulation.

89. Liu W, Zhang Q, Zhang J, Pan W, Zhao J, Xu Y. Long non-coding RNA MALAT1 contributes to cell apoptosis by sponging miR-124 in Parkinson disease. Cell Biosci. 7, 19 (2017).

90. Yang $\mathrm{H}$, Liang $\mathrm{N}$, Wang $\mathrm{M}$ et al. Long noncoding RNA MALAT-1 is a novel inflammatory regulator in human systemic lupus erythematosus. Oncotarget 8(44), 77400-77406 (2017).

91. Zhang F, Wu L, Qian J et al. Identification of the long noncoding RNA NEAT1 as a novel inflammatory regulator acting through MAPK pathway in human lupus. J. Autoimmun. 75, 96-104 (2016).

92. Enghard P, Humrich JY, Rudolph B et al. $\mathrm{CXCR} 3^{+} \mathrm{CD} 4^{+} \mathrm{T}$ cells are enriched in inflamed kidneys and urine and provide a new biomarker for acute nephritis flares in systemic lupus erythematosus patients. Arthritis Rheum. 60(1), 199-206 (2009).

93. Masutani K, Akahoshi M, Tsuruya K et al. Predominance of Th1 immune response in diffuse proliferative lupus nephritis. Arthritis Rheum. 44(9), 2097-2106 (2001).

94. Steinmetz OM, Turner JE, Paust HJ et al. CXCR3 mediates renal Th1 and Th17 immune response in murine lupus nephritis. J. Immunol. 183(7), 4693-4704 (2009).

95. Teramoto K, Negoro N, Kitamoto K et al. Microarray analysis of glomerular gene expression in murine lupus nephritis. J. Pharmacol. Sci. 106(1), 56-67 (2008).

96. Wu Y, Zhang F, Ma J et al. Association of large intergenic noncoding RNA expression with disease activity and organ damage in systemic lupus erythematosus. Arthritis Res. Ther. 17, 131 (2015).

97. Suarez-Gestal M, Calaza M, Endreffy E et al. Replication of recently identified systemic lupus erythematosus genetic associations: a case-control study. Arthritis Res. Ther. 11(3), R69 (2009).

98. Suravajhala P, Kogelman LJ, Mazzoni G, Kadarmideen HN. Potential role of lncRNA cyp2c91-protein interactions on diseases of the immune system. Front. Genet. 6, 255 (2015).

99. Wang J, Syrett CM, Kramer MC, Basu A, Atchison ML, Anguera MC. Unusual maintenance of X chromosome inactivation predisposes female lymphocytes for increased expression from the inactive X. Proc. Natl Acad. Sci. USA 113(14), E2029-E2038 (2016).

100. Song J, Kim D, Han J, Kim Y, Lee M, Jin EJ. PBMC and exosome-derived Hotair is a critical regulator and potent marker for rheumatoid arthritis. Clin. Exp. Med. 15(1), 121-126 (2015). 
101. Zhang C, Wang P, Jiang P et al. Upregulation of IncRNA HOTAIR contributes to IL-1 $\beta$-induced MMP overexpression and chondrocytes apoptosis in temporomandibular joint osteoarthritis. Gene 586(2), 248-253 (2016).

102. Zhang HJ, Wei QF, Wang SJ et al. LncRNA HOTAIR alleviates rheumatoid arthritis by targeting miR-138 and inactivating NF- $\mathrm{KB}$ pathway. Int. Immunopharmacol. 50, 283-290 (2017).

103. Stuhlmüller B, Kunisch E, Franz J et al. Detection of oncofetal H19 RNA in rheumatoid arthritis synovial tissue. Am. J. Pathol. 163(3), 901-911 (2003).

104. Hayashida T, Eversole-Cire P, Jones PA, Sasaki H. Imprinted genes are up-regulated by growth arrest in embryonic fibroblasts. J. Biochem. 122(5), 901-903 (1997).

105. Spurlock CF 3rd, Gass HMT, Bryant CJ, Wells BC, Olsen NJ, Aune TM. Methotrexate-mediated inhibition of nuclear factor $\mathrm{\kappa B}$ activation by distinct pathways in T cells and fibroblast-like synoviocytes. Rheumatology (Oxford) 54(1), 178-187 (2015).

106. Peng QL, Zhang YM, Yang HB, Shu XM, Lu X, Wang GC. Transcriptomic profiling of long non-coding RNAs in dermatomyositis by microarray analysis. Sci. Rep. 6, 32818 (2016).

107. Wang Z, Jinnin M, Nakamura $\mathrm{K}$ et al. Long non-coding RNA TSIX is upregulated in scleroderma dermal fibroblasts and controls collagen mRNA stabilization. Exp. Dermatol. 25(2), 131-136 (2016).

108. Griseri P, Pages G. Regulation of the mRNA half-life in breast cancer. World J. Clin. Oncol. 5(3), 323-334 (2014).

109. Lafyatis R. Transforming growth factor $\beta$ - at the centre of systemic sclerosis. Nat. Rev. Rheumatol. 10(12), 706-719 (2014).

110. Pachera E, Assassi S, Salazar Cintora G et al. OP0284 long noncoding RNA MIR503HG is a novel factor in the pathogenesis of systemic sclerosis. Ann. Rheum. Dis. 74(S2), 180-181 (2015).

111. Shi H, Cao N, Pu Y, Xie L, Zheng L, Yu C. Long non-coding RNA expression profile in minor salivary gland of primary Sjogren's syndrome. Arthritis Res. Ther. 18(1), 109 (2016).

112. Collier SP, Henderson MA, Tossberg JT, Aune TM. Regulation of the Th1 genomic locus from Ifng through Tmevpg1 by T-bet. J. Immunol. 193(8), 3959-3965 (2014).

113. Salzman J. Circular RNA expression: its potential regulation and function. Trends Genet. 32(5), 309-316 (2016).

-. A summary of circRNA, focus on its potential regulation and function.

114. Sanger HI, Klotz G, Riesner D, Gross HJ, Ak K. Viroids are single-stranded covalently closed circular RNA molecules existing as highly base-paired rod-like structures. Proc. Natl Acad. Sci. USA 73(11), 3852-3856 (1976).

115. Petkovic S, Muller S. RNA circularization strategies in vivo and in vitro. Nucleic Acids Res. 43(4), 2454-2465 (2015).

116. Rybak-Wolf A, Stottmeister C, Glazar P et al. Circular RNAs in the mammalian brain are highly abundant, conserved, and dynamically expressed. Mol. Cell 58(5), 870-885 (2015).

117. Shan K, Liu C, Liu BH. Circular noncoding RNA HIPK3 mediates retinal vascular dysfunction in diabetes mellitus. Circulation 136(17), 1629-1642 (2017).

118. Tang CM, Zhang M, Huang L et al. CircRNA_000203 enhances the expression of fibrosis-associated genes by derepressing targets of miR-26b-5p, Col1a2 and CTGF, in cardiac fibroblasts. Sci. Rep. 7, 40342 (2017).

119. Holdt LM, Stahringer A, Sass K et al. Circular non-coding RNA ANRIL modulates ribosomal RNA maturation and atherosclerosis in humans. Nat. Commun. 7, 12429 (2016).

120. Luan J, Jiao C, Kong W et al. CircHLA-C plays an important role in lupus nephritis by sponging miR-150. Mol. Ther. Nucleic Acids 10, $245-253$ (2018).

121. Ouyang Q, Huang Q, Jiang Z, Zhao J, Shi GP, Yang M. Using plasma circRNA_002453 as a novel biomarker in the diagnosis of lupus nephritis. Mol. Immunol. 101, 531-538 (2018).

122. Li LJ, Zhu ZW, Zhao W et al. Circular RNA expression profile and potential function of hsa_circ_0045272 in systemic lupus erythematosus. Immunology 155(1), 137-149 (2018).

123. Ouyang Q, Wu J, Jiang Z et al. Microarray expression profile of circular RNAs in peripheral blood mononuclear cells from rheumatoid arthritis patients. Cell Physiol. Biochem. 42(2), 651-659 (2017).

124. Luo Q, Zhang L, Li X et al. Identification of circular RNAs hsa_circ_0044235 in peripheral blood as novel biomarkers for rheumatoid arthritis. Clin. Exp. Immunol. 194(1), 118-124 (2018).

125. Li B, Li N, Zhang L et al. Hsa_circ_0001859 regulates ATF2 expression by functioning as an MiR-204/211 sponge in human rheumatoid arthritis. J. Immunol. Res. 2018, 9412387 (2018).

126. Iwasaki YW, Siomi MC, Siomi H. PIWI-interacting RNA: its biogenesis and functions. Annu. Rev. Biochem. 84, 405-433 (2015).

127. Plestilova L, Neidhart M, Russo G et al. Expression and regulation of PIWIL-proteins and PIWI-interacting RNAs in rheumatoid arthritis. PLoS ONE 11(11), e0166920 (2016). 
(

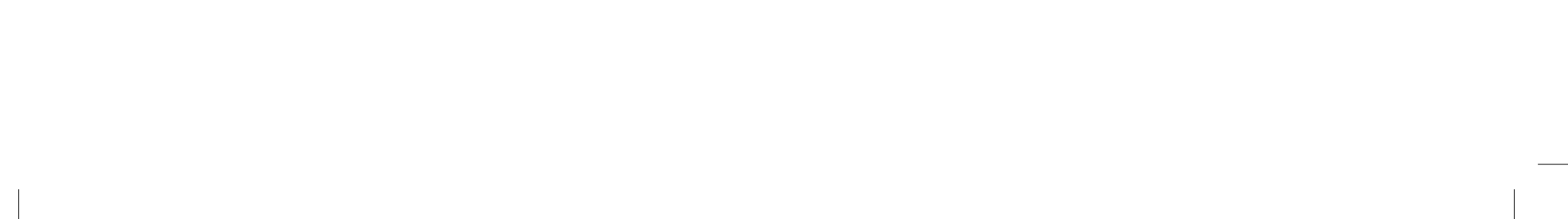

